\title{
Pullback Attractor for Nonautonomous Primitive Equations of Large-Scale Ocean and Atmosphere Dynamics
}

\author{
Kun $\mathrm{Li}^{1}$ and Fang $\mathrm{Li}^{2}$ \\ ${ }^{1}$ Department of Basic, Henan Mechanical and Electrical Engineering College, Xinxiang 453003, China \\ ${ }^{2}$ Department of Mathematics, Nanjing University, Nanjing 210093, China
}

Correspondence should be addressed to Fang Li; lifang101216@126.com

Received 9 April 2013; Accepted 18 June 2013

Academic Editor: Grzegorz Lukaszewicz

Copyright (c) $2013 \mathrm{~K}$. Li and F. Li. This is an open access article distributed under the Creative Commons Attribution License, which permits unrestricted use, distribution, and reproduction in any medium, provided the original work is properly cited.

We consider the existence of $(V, V)$-pullback attractor for nonautonomous primitive equations of large-scale ocean and atmosphere dynamics in a three-dimensional bounded cylindrical domain by verifying pullback $\mathscr{D}$ condition.

\section{Introduction}

This paper is concerned with the existence of pullback attractor for the following nonautonomous primitive equations of large-scale ocean and atmosphere dynamics:

$$
\begin{aligned}
& \frac{\partial v}{\partial t}+(v \cdot \nabla) v-\left(\int_{-h}^{z} \nabla \cdot v(x, y, \zeta, t) d \zeta\right) \frac{\partial v}{\partial z}+\nabla p_{s}(x, y, t) \\
&-\int_{0}^{z} \nabla T(x, y, \zeta, t) d \zeta+\frac{1}{\mathrm{Ro}} f v^{\perp}+L_{1} v=0 \\
& \frac{\partial T}{\partial t}+v \cdot \nabla T-\left(\int_{-h}^{z} \nabla \cdot v(x, y, \zeta, t) d \zeta\right) \frac{\partial T}{\partial z}+L_{2} T=Q
\end{aligned}
$$

with the following boundary conditions:

$$
\begin{array}{rlrl}
\left.\frac{\partial v}{\partial z}\right|_{z=0}=0, & \left.\frac{\partial v}{\partial z}\right|_{z=-h}=0, \\
\left.v \cdot \vec{n}\right|_{\Gamma_{l}}=0, & \frac{\partial v}{\partial \vec{n}} \times\left.\vec{n}\right|_{\Gamma_{l}}=0, \\
\left.\left(\frac{1}{R t_{2}} \frac{\partial T}{\partial z}+\alpha T\right)\right|_{z=0}=0, & \left.\frac{\partial T}{\partial z}\right|_{z=-h}=0, & \left.\frac{\partial T}{\partial \vec{n}}\right|_{\Gamma_{l}}=0,
\end{array}
$$

and the initial data

$$
\begin{aligned}
v(x, y, z, \tau) & =v_{0}(x, y, z), \\
T(x, y, z, \tau) & =T_{0}(x, y, z),
\end{aligned}
$$

in the three-dimensional bounded cylindrical domain

$$
\Omega=M \times(-h, 0),
$$

where $M$ is a bounded domain in $\mathbb{R}^{2}$ with smooth boundary $\partial M$ and $h$ is a positive constant, the unknown functions $v(x, y, z, t)=\left(v_{1}(x, y, z, t), v_{2}(x, y, z, t)\right), T(x, y$, $z, t), p_{s}(x, y, t)$ denote the horizontal velocity, the temperature, and the pressure at $z=0$, respectively, $v^{\perp}=$ $\left(-v_{2}, v_{1}\right), f=f_{0}+\beta y$ is the Coriolis parameter, Ro is the Rossby number which measures the significant influence of the Earth's rotation to the dynamical behaviour of the ocean, $Q(x, y, z, t)$ is a given heat source, $\Gamma_{l}=\{(x, y, z) \in \bar{\Omega}$ : $(x, y) \in \partial M,-h \leq z \leq 0\}$ denotes the lateral boundary of $\Omega, \vec{n}$ is the normal vector to $\Gamma_{l}$, and $\alpha$ is a positive constant related with the turbulent heating on the surface of the ocean. The viscosity and the heat diffusion operators $L_{1}$ and $L_{2}$ are given by

$$
\begin{aligned}
& L_{1}=-\frac{1}{\operatorname{Re}_{1}} \Delta-\frac{1}{\mathrm{Re}_{2}} \frac{\partial^{2}}{\partial z^{2}}, \\
& L_{2}=-\frac{1}{\mathrm{Rt}_{1}} \Delta-\frac{1}{\mathrm{Rt}_{2}} \frac{\partial^{2}}{\partial z^{2}},
\end{aligned}
$$

where $\mathrm{Re}_{1}, \mathrm{Re}_{2}$ are positive constants representing the horizontal and vertical Reynolds numbers, respectively, and $\mathrm{Rt}_{1}, \mathrm{Rt}_{2}$ are positive constants which stand for the horizontal and vertical eddy diffusivity, respectively. For the sake of 
simplicity, let $\nabla=\left(\partial_{x}, \partial_{y}\right)$ be the horizontal gradient operator, and let $\Delta=\partial_{x}^{2}+\partial_{y}^{2}$ be the horizontal Laplacian.

Nonautonomous equations appear in many applications in the natural sciences, and so they are of great importance and interest. The long-time behavior of solutions of such equations has been studied extensively in recent years (see [1-12], etc.). The first attempt was to extend the notion of global attractor to the nonautonomous case, leading to the concept of the so-called uniform attractor (see [13]). It is remarkable that the conditions ensuring the existence of the uniform attractor is parallel those for the autonomous case. However, one disadvantage of the uniform attractor is that it does not need to be "invariant," unlike the global attractor for autonomous systems. Moreover, it is well known that the trajectories may be unbounded for many nonautonomous systems when the time tends to infinity, and there does not exist a uniform attractor for these systems. In order to overcome this drawback, the concept of pullback attractor has been introduced for the nonautonomous case. The theory of pullback attractors has been developed for both nonautonomous and random dynamical systems, and it has been shown to be very useful in the understanding of the dynamics of nonautonomous dynamical systems (see [6]).

Large-scale dynamics of oceans and atmosphere is governed by the primitive equations which are derived from the Navier-Stokes equations with rotation, coupled to thermodynamics and salinity diffusion-transport equations, which account for the buoyancy forces and stratification effects under the Boussinesq approximation. Moreover, due to the shallowness of the oceans and atmosphere, that is, the depth of the fluid layer is very small in comparison to the radius of the Earth, the vertical large-scale motion in the oceans and atmosphere is much smaller than the horizontal one, which in turn leads to modeling the vertical motion by the hydrostatic balance. As a result, one can obtain the system (1)-(4), which is known as the primitive equations for largescale oceans and atmosphere dynamics (see [14-18]). In the case of ocean dynamics, we know that one has to add the diffusion-transport equation of the salinity to the system (1)-(4), but we will omit it here in order to simplify our mathematical presentation. However, we emphasize that our results are equally valid when the salinity effects are taken into account.

In recent years, the primitive equations of the atmosphere, the ocean, and the coupled atmosphere-ocean have been extensively studied from the mathematical point of view (see [14-16, 19-26], etc.). The mathematical framework of the primitive equations of the ocean was formulated, and the existence of weak solutions was proved by Lions et al. in [15]. In [16], the authors proved the existence and the uniqueness of local in time strong solutions of the primitive equations of the ocean. The global existence of strong solutions of the three-dimensional primitive equations of large-scale ocean, by assuming that the initial data are small enough, and the local existence of strong solutions of the three-dimensional primitive equations of large-scale ocean for all initial data were proved by Guillén-González et al. in [27]. In [19], the authors proved the maximum principles for the primitive equations of the atmosphere. The existence and uniqueness of strong solutions, global in time, to the primitive equations in thin domains for a large set of initial data whose sizes depend inversely on the thickness were established in [23]. In [28], Temam and Ziane considered the local existence of strong solutions for the primitive equations of the atmosphere, the ocean, and the coupled atmosphereocean. Asymptotic analysis of the primitive equations under the small depth assumption was established in [24]. In [22], the authors proved the existence of weak solutions and trajectory attractors for the moist atmospheric equations in geophysics. The existence and uniqueness of global strong solutions for the initial boundary value problem of the threedimensional viscous primitive equations of large-scale ocean were established by the authors in [14]. In [20], the authors considered the long-time dynamics of the primitive equations of large-scale atmosphere and obtained a weakly compact global attractor $\mathscr{A}$ which captures all the trajectories with respect to $V$-weak topology. Under the assumption of the initial data $\left(v_{0}, T_{0}\right) \in V \cap\left(H^{2}(\Omega)\right)^{3}$, the existence of compact global attractor in $V$ for the primitive equations of large-scale atmosphere was established by the elliptic regularity theory in [21]. In [26], the authors proved the global existence and uniqueness of $z$-weak solutions when the initial conditions satisfy some regularity. The existence of global attractors in $V$ and $\left(H^{2}(\Omega)\right)^{3}$ for three-dimensional viscous primitive equations of large-scale atmosphere in logpressure coordinates was established in [29]. In [30], the authors proved the existence of $(V, V)$-global attractor for the primitive equations of large-scale atmosphere and ocean dynamics by use of the Aubin-Lions compactness theorem. Attractor is an important concept in the study of infinitedimensional dynamical systems; however, the existence of pullback attractor for the three-dimensional nonautonomous viscous primitive equations of large-scale atmosphere and ocean dynamics remains unsolvable. In this paper, we prove the existence of pullback attractor for nonautonomous equations (1)-(5) by verifying pullback $\mathscr{D}$ condition. This paper is organized as follows. In Section 2, we introduce the mathematical framework of the system (1)-(5) and recall some auxiliary lemmas used to give some a priori estimates of strong solutions for (1)-(5) in Section 3, which are used to obtain the existence of $(V, V)$-pullback absorbing set. Finally, in Section 4, we are devoted to proving the existence of $(V, V)$ pullback attractor for the three dimensional viscous primitive equations of large-scale ocean and atmosphere dynamics by verifying pullback $\mathscr{D}$ condition.

Throughout this paper, let $X$ be a Banach space endowed with the norm $\|\cdot\|_{X}$, let $\|u\|_{p}$ be the $L^{p}(\Omega)$-norm of $u$, and let $C$ be positive constants, which may be different from line to line.

\section{Preliminaries}

2.1. Functional Spaces and Some Lemmas. To study problem (1)-(5), we introduce some function spaces. Let

$$
\mathscr{V}_{1}=\left\{v \in\left(C^{\infty}(\Omega)\right)^{2}:\left.\frac{\partial v}{\partial z}\right|_{z=0}=0,\left.\frac{\partial v}{\partial z}\right|_{z=-h}=0,\right.
$$




$$
\begin{gathered}
\left.v \cdot \vec{n}\right|_{\Gamma_{l}}=0, \frac{\partial v}{\partial \vec{n}} \times\left.\vec{n}\right|_{\Gamma_{l}}=0, \\
\left.\int_{-h}^{0} \nabla \cdot v(x, y, \zeta) d \zeta=0\right\}, \\
\mathscr{V}_{2}=\left\{T \in C^{\infty}(\Omega):\left.\left(\frac{1}{\mathrm{Rt}_{2}} \frac{\partial T}{\partial z}+\alpha T\right)\right|_{z=0}=0,\right. \\
\left.\left.\frac{\partial T}{\partial z}\right|_{z=-h}=0,\left.\frac{\partial T}{\partial \vec{n}}\right|_{\Gamma_{l}}=0\right\} .
\end{gathered}
$$

Denote the closure of $\mathscr{V}_{1}, \mathscr{V}_{2}$ by $V_{1}, V_{2}$ with respect to the following norms, respectively, defined as follows:

$$
\begin{aligned}
\|v\|^{2}= & \frac{1}{\mathrm{Re}_{1}} \int_{\Omega}|\nabla v|^{2} d x d y d z+\frac{1}{\mathrm{Re}_{2}} \int_{\Omega}\left|\partial_{z} v\right|^{2} d x d y d z, \\
\|T\|^{2}= & \frac{1}{\mathrm{Rt}_{1}} \int_{\Omega}|\nabla T|^{2} d x d y d z+\frac{1}{\mathrm{Rt}_{2}} \int_{\Omega}\left|\partial_{z} T\right|^{2} d x d y d z \\
& +\alpha \int_{M}|T(z=0)|^{2} d x d y,
\end{aligned}
$$

for any $v \in \mathscr{V}_{1}, T \in \mathscr{V}_{2}$, and let $H_{1}=$ the closure of $\mathscr{V}_{1}$ with respect to the norm $\left(L^{2}(\Omega)\right)^{2}, V=V_{1} \times V_{2}, H=H_{1} \times L^{2}(\Omega)$.

Now, we recall some lemmas used in the sequel.

Lemma 1 (see $[13,31])$. Let $y(t), a(t), h(t)$ be three positive locally integrable functions on $\left[t_{0}, \infty\right)$, and for some $r>0$ and all $t \geq t_{0}$, the following inequalities hold:

$$
\begin{gathered}
y^{\prime} \leq a(t) y+h(t), \\
\int_{t}^{t+r} y(\tau) d \tau \leq X, \quad \int_{t}^{t+r} a(\tau) d \tau \leq A, \\
\int_{t}^{t+r} h(\tau) d \tau \leq B .
\end{gathered}
$$

Then,

$$
y(t) \leq\left(\frac{X}{r}+B\right) e^{A},
$$

for all $t \geq t_{0}+r$.

Lemma 2 (see [14]) (Minkowski inequality). Let $(X, \mu)$, $(Y, v)$ be two measure spaces and $f(x, y)$ be a measurable function about $\mu \times v$ on $X \times Y$. If for a.e. $y \in Y, f(\cdot, y) \in$ $L^{p}(X, \mu)(1 \leq p \leq \infty)$, and $\int_{Y}\|f(\cdot, y)\|_{L^{p}(X, \mu)} d \nu(y)<\infty$, then

$$
\left\|\int_{Y} f(\cdot, y) d \nu(y)\right\|_{L^{p}(X, \mu)} \leq \int_{Y}\|f(\cdot, y)\|_{L^{p}(X, \mu)} d \nu(y) .
$$

2.2. New Formulation. In this subsection, we divide (1) into two systems with respect to $\bar{v}$ and $\widetilde{v}$ defined by

$$
\begin{gathered}
\bar{v}(x, y)=\frac{1}{h} \int_{-h}^{0} v(x, y, \zeta) d \zeta, \\
\widetilde{v}=v-\bar{v} .
\end{gathered}
$$

Taking the average of (1) in the $z$ direction over the interval $(-h, 0)$ and using the boundary conditions (3), we obtain

$$
\begin{aligned}
\frac{\partial \bar{v}}{\partial t}+ & (\bar{v} \cdot \nabla) \bar{v}+\overline{(\widetilde{v} \cdot \nabla) \widetilde{v}+(\nabla \cdot \widetilde{v}) \widetilde{v}}+\nabla p_{s}(x, y, t)-\frac{1}{\mathrm{Re}_{1}} \Delta \bar{v} \\
& +\frac{1}{\operatorname{Ro}} f \bar{v}^{\perp}-\overline{\int_{0}^{z} \nabla T(x, y, \zeta, t) d \zeta}=0,
\end{aligned}
$$

with the boundary conditions

$$
\nabla \cdot \bar{v}=0,\left.\quad \bar{v} \cdot \vec{n}\right|_{\Gamma_{l}}=0, \quad \frac{\partial \bar{v}}{\partial \vec{n}} \times\left.\vec{n}\right|_{\Gamma_{l}}=0 .
$$

Subtracting (14) from (1), we get

$$
\begin{aligned}
\frac{\partial \widetilde{v}}{\partial t}+ & (\widetilde{v} \cdot \nabla) \widetilde{v}-\left(\int_{-h}^{z} \nabla \cdot \widetilde{v}(x, y, \zeta, t) d \zeta\right) \frac{\partial \widetilde{v}}{\partial z} \\
& +(\widetilde{v} \cdot \nabla) \bar{v}-\overline{(\widetilde{v} \cdot \nabla) \widetilde{v}+(\nabla \cdot \widetilde{v}) \widetilde{v}} \\
& +(\bar{v} \cdot \nabla) \widetilde{v}-\int_{0}^{z} \nabla T(x, y, \zeta, t) d \zeta \\
& +\overline{\int_{0}^{z} \nabla T(x, y, \zeta, t) d \zeta}+\frac{1}{\operatorname{Ro}} f \widetilde{v}^{\perp}+L_{1} \widetilde{v}=0,
\end{aligned}
$$

with the boundary conditions

$$
\begin{array}{cc}
\left.\frac{\partial \widetilde{v}}{\partial z}\right|_{\Gamma_{u}}=0, & \left.\frac{\partial \widetilde{v}}{\partial z}\right|_{\Gamma_{b}}=0, \\
\left.\widetilde{v} \cdot \vec{n}\right|_{\Gamma_{l}}=0, & \frac{\partial \widetilde{v}}{\partial \vec{n}} \times\left.\vec{n}\right|_{\Gamma_{l}}=0 .
\end{array}
$$

\section{Some a Priori Estimates of Strong Solutions}

3.1. The Well Posedness of Strong Solutions. We start with the following general existence and uniqueness of solutions which can be obtained by the standard Fatou-Galerkin methods [31] and the similar methods established in [14]. Here, we only state the result as follows.

Theorem 3. Suppose that $Q \in L_{\mathrm{loc}}^{2}\left(\mathbb{R} ; L^{2}(\Omega)\right)$. Then for any $\tau \in \mathbb{R}$, any initial data $\left(v_{0}, T_{0}\right) \in V$, and any $\mathscr{T}>0$, there exists a unique solution $(v, T) \in C([0, \mathscr{T}] ; V)$ of (1)(5). Furthermore, the solution is continuous with respect to the initial data in $V$.

By Theorem 3, we can define a family of continuous processes $\{U(t, \tau):-\infty<\tau \leq t<\infty\}$ in $V$ by

$$
U(t, \tau)\left(v_{0}, T_{0}\right)=(v(t), T(t)):=\left(v\left(t ; \tau, v_{0}\right), T\left(t ; \tau, T_{0}\right)\right),
$$

$\forall t \geq \tau$, 
where $(v(t), T(t))$ is the solution of (1)-(5) with initial data $(v(\tau), T(\tau))=\left(v_{0}, T_{0}\right) \in V$. That is, a family of mappings $U(t, \tau): V \rightarrow V$ satisfies

$$
\begin{gathered}
U(\tau, \tau)=\text { id (identity), } \\
U(t, \tau)=U(t, r) U(r, \tau) \quad \forall \tau \leq r \leq t .
\end{gathered}
$$

3.2. Some a Priori Estimates about Strong Solutions. In this subsection, assume that $0<\sigma \leq \min \left\{1 /\left(4 \mathrm{Rt}_{2} h^{2}+\right.\right.$ $\left.(4 h / \alpha)), 1 / 2 C_{M} \operatorname{Re}_{1}\right\}$ and $\sup _{r \in \mathbb{R}} \int_{r-1}^{r}\|Q(s)\|_{2}^{2} d s<\infty$; we give some a priori estimates of strong solutions which imply the existence of pullback absorbing set.

3.2.1. $L^{2}(\Omega)$ Estimate of $T$. Taking the inner product of (2) with $T$ in $L^{2}(\Omega)$ and combining the boundary conditions (4), we get

$$
\begin{aligned}
& \frac{1}{2} \frac{d}{d t}\|T\|_{2}^{2}+\|T\|^{2} \\
& =-\int_{\Omega}\left[v \cdot \nabla T-\left(\int_{-h}^{z} \nabla \cdot v(x, y, \zeta, t) d \zeta\right)\right. \\
& \left.\quad \times \frac{\partial T}{\partial z}\right] T d x d y d z+\int_{\Omega} Q T d x d y d z \\
& =\int_{\Omega} Q T d x d y d z .
\end{aligned}
$$

Integrating by parts and using the boundary conditions (3), we obtain

$$
\int_{\Omega}\left[v \cdot \nabla T-\left(\int_{-h}^{z} \nabla \cdot v(x, y, \zeta, t) d \zeta\right) \frac{\partial T}{\partial z}\right] T d x d y d z=0 .
$$

Since

$$
\|T\|_{2}^{2} \leq 2 h\|T(z=0)\|_{L^{2}(M)}^{2}+2 h^{2}\left\|\partial_{z} T\right\|_{2}^{2},
$$

it is implied that

$$
\begin{aligned}
\frac{\|T\|_{2}^{2}}{2 \mathrm{Rt}_{2} h^{2}+(2 h / \alpha)} \leq & \frac{2 h\|T(z=0)\|_{L^{2}(M)}^{2}+2 h^{2}\left\|\partial_{z} T\right\|_{2}^{2}}{2 \mathrm{Rt}_{2} h^{2}+(2 h / \alpha)} \\
\leq & \frac{1}{\mathrm{Rt}_{2}} \int_{\Omega}\left|\partial_{z} T\right|^{2} d x d y d z \\
& +\alpha \int_{M}|T(z=0)|^{2} d x d y \\
\leq & \|T\|^{2} .
\end{aligned}
$$

It follows from (20) and (23) that

$$
\frac{d}{d t}\|T\|_{2}^{2}+\|T\|^{2} \leq\left(2 \mathrm{Rt}_{2} h^{2}+\frac{2 h}{\alpha}\right)\|Q\|_{2}^{2} .
$$

Using (23) again, we get

$$
\frac{d}{d t}\|T\|_{2}^{2}+\frac{\|T\|_{2}^{2}}{2 \mathrm{Rt}_{2} h^{2}+(2 h / \alpha)} \leq\left(2 \mathrm{Rt}_{2} h^{2}+\frac{2 h}{\alpha}\right)\|Q\|_{2}^{2} .
$$

Therefore, we deduce from (24) and (25) that

$$
\begin{aligned}
& \frac{d}{d t}\left(e^{\sigma t}\|T\|_{2}^{2}\right)+\frac{1}{2} e^{\sigma t}\|T\|^{2} \\
& =e^{\sigma t} \frac{d}{d t}\|T\|_{2}^{2}+\sigma e^{\sigma t}\|T\|_{2}^{2}+\frac{1}{2} e^{\sigma t}\|T\|^{2} \\
& \leq\left(\sigma-\frac{1}{4 \mathrm{Rt}_{2} h^{2}+(4 h / \alpha)}\right) e^{\sigma t}\|T\|_{2}^{2} \\
& +\left(2 \mathrm{Rt}_{2} h^{2}+\frac{2 h}{\alpha}\right) e^{\sigma t}\|\mathrm{Q}\|_{2}^{2} \\
& \leq\left(2 \mathrm{Rt}_{2} h^{2}+\frac{2 h}{\alpha}\right) e^{\sigma t}\|Q\|_{2}^{2} \text {. }
\end{aligned}
$$

Integrating (26) from $\tau$ to $t$, we obtain

$$
\begin{aligned}
& e^{\sigma t}\|T(t)\|_{2}^{2}+\frac{1}{2} \int_{\tau}^{t} e^{\sigma s}\|T(s)\|^{2} d s \\
& \leq e^{\sigma \tau}\left\|T_{0}\right\|_{2}^{2}+\left(2 \mathrm{Rt}_{2} h^{2}+\frac{2 h}{\alpha}\right) \int_{\tau}^{t} e^{\sigma s}\|Q(s)\|_{2}^{2} d s \\
& \leq e^{\sigma \tau}\left\|T_{0}\right\|_{2}^{2}+\left(2 \mathrm{Rt}_{2} h^{2}+\frac{2 h}{\alpha}\right) \int_{-\infty}^{t} e^{\sigma s}\|Q(s)\|_{2}^{2} d s \\
& \leq e^{\sigma \tau}\left\|T_{0}\right\|_{2}^{2}+e^{\sigma t}\left(2 \mathrm{Rt}_{2} h^{2}+\frac{2 h}{\alpha}\right) \\
& \quad \times\left(1+\sigma^{-1}\right) \sup _{t \in \mathbb{R}} \int_{t}^{t+1}\|Q(s)\|_{2}^{2} d s,
\end{aligned}
$$

where we use the inequality

$$
\begin{aligned}
\int_{-\infty}^{t} & e^{\sigma s}\|Q(s)\|_{2}^{2} d s \\
= & \int_{t-1}^{t} e^{\sigma s}\|Q(s)\|_{2}^{2} d s+\int_{t-2}^{t-1} e^{\sigma s}\|Q(s)\|_{2}^{2} d s \\
& +\int_{t-3}^{t-2} e^{\sigma s}\|Q(s)\|_{2}^{2} d s+\int_{t-4}^{t-3} e^{\sigma s}\|Q(s)\|_{2}^{2} d s+\cdots \\
& +\int_{t-n}^{t-n+1} e^{\sigma s}\|Q(s)\|_{2}^{2} d s+\cdots \\
\leq & \left(e^{\sigma t}+e^{\sigma(t-1)}+e^{\sigma(t-2)}+e^{\sigma(t-3)}+\cdots\right) \\
& \times \sup _{r \in \mathbb{R}} \int_{r}^{r+1}\|Q(s)\|_{2}^{2} d s \\
\leq & \frac{e^{\sigma t}}{1-e^{-\sigma}} \sup _{r \in \mathbb{R}} \int_{r}^{r+1}\|Q(s)\|_{2}^{2} d s \\
\leq & \left(1+\sigma^{-1}\right) e^{\sigma t} \sup _{r \in \mathbb{R}} \int_{r}^{r+1}\|Q(s)\|_{2}^{2} d s .
\end{aligned}
$$


That is,

$$
\begin{aligned}
\|T(t)\|_{2}^{2} \leq & e^{\sigma(\tau-t)}\left\|T_{0}\right\|_{2}^{2}+\left(2 \mathrm{Rt}_{2} h^{2}+\frac{2 h}{\alpha}\right) \\
& \times\left(1+\sigma^{-1}\right) \sup _{t \in \mathbb{R}} \int_{t}^{t+1}\|Q(s)\|_{2}^{2} d s .
\end{aligned}
$$

Let $\tau<t-1$, for any $r \in[\tau, t-1]$; integrating (24) from $r$ to $r+1$ and combining with (29), we obtain

$$
\begin{aligned}
\int_{r}^{r+1} & \|T(s)\|^{2} d s \\
\leq & \left(2 \mathrm{Rt}_{2} h^{2}+\frac{2 h}{\alpha}\right) \int_{r}^{r+1}\|Q(s)\|_{2}^{2} d s+\|T(r)\|_{2}^{2} \\
\leq & e^{\sigma(\tau-r)}\left\|T_{0}\right\|_{2}^{2}+\left(2 \mathrm{Rt}_{2} h^{2}+\frac{2 h}{\alpha}\right) \\
& \times\left(2+\sigma^{-1}\right) \sup _{t \in \mathbb{R}} \int_{t}^{t+1}\|Q(s)\|_{2}^{2} d s .
\end{aligned}
$$

3.2.2. $H_{1}$ Estimates of $v$. Multiplying (1) by $v$ and integrating by parts over $\Omega$ and using the boundary conditions (3), we deduce

$$
\begin{aligned}
& \frac{1}{2} \frac{d}{d t}\|v\|_{2}^{2}+\|v\|^{2} \\
& \quad=\int_{\Omega}\left(\int_{0}^{z} \nabla T(x, y, \zeta, t) d \zeta\right) \cdot v d x d y d z \\
& \quad \leq h\|T\|_{2}\|\nabla v\|_{2} \\
& \quad \leq \frac{1}{2 \operatorname{Re}_{1}} \int_{\Omega}|\nabla v|^{2} d x d y d z+\frac{\operatorname{Re}_{1} h^{2}\|T\|_{2}^{2}}{2} .
\end{aligned}
$$

That is,

$$
\frac{d}{d t}\|v\|_{2}^{2}+\|v\|^{2} \leq \operatorname{Re}_{1} h^{2}\|T\|_{2}^{2}
$$

It follows from $\|v\|_{2}^{2} \leq C_{M}\|\nabla v\|_{2}^{2}$ that

$$
\frac{d}{d t}\|v\|_{2}^{2}+\frac{1}{C_{M} \operatorname{Re}_{1}}\|v\|_{2}^{2} \leq \operatorname{Re}_{1} h^{2}\|T\|_{2}^{2} .
$$

Thanks to (32) and (33), we get

$$
\begin{aligned}
& \frac{d}{d t}\left(e^{\sigma t}\|v\|_{2}^{2}\right)+\frac{1}{2} e^{\sigma t}\|v\|^{2} \\
& \quad=e^{\sigma t} \frac{d}{d t}\|v\|_{2}^{2}+\sigma e^{\sigma t}\|v\|_{2}^{2}+\frac{1}{2} e^{\sigma t}\|v\|^{2} \\
& \quad \leq\left(\sigma-\frac{1}{2 C_{M} \operatorname{Re}_{1}}\right) e^{\sigma t}\|v\|_{2}^{2}+\operatorname{Re}_{1} h^{2} e^{\sigma t}\|T\|_{2}^{2}
\end{aligned}
$$

Integrating (34) over $[\tau, t]$ and using (23) and (27), we have

$$
\begin{aligned}
& e^{\sigma t}\|v(t)\|_{2}^{2}+\frac{1}{2} \int_{\tau}^{t} e^{\sigma s}\|v\|^{2} \\
& \leq e^{\sigma \tau}\left\|v_{0}\right\|_{2}^{2}+\operatorname{Re}_{1} h^{2} \int_{\tau}^{t} e^{\sigma s}\|T(s)\|_{2}^{2} d s \\
& \leq e^{\sigma \tau}\left\|v_{0}\right\|_{2}^{2}+\operatorname{Re}_{1} h^{2}\left(2 \mathrm{Rt}_{2} h^{2}+\frac{2 h}{\alpha}\right) \\
& \quad \times \int_{\tau}^{t} e^{\sigma s}\|T(s)\|^{2} d s \\
& \leq e^{\sigma \tau}\left\|v_{0}\right\|_{2}^{2}+2\left(\operatorname{Re}_{1} h^{2}\left(2 \mathrm{Rt}_{2} h^{2}+\frac{2 h}{\alpha}\right)\right) \\
& \quad \times\left(e^{\sigma \tau}\left\|T_{0}\right\|_{2}^{2}+e^{\sigma t} \rho_{1}\right),
\end{aligned}
$$

where

$$
\rho_{1}=\left(2 \mathrm{Rt}_{2} h^{2}+\frac{2 h}{\alpha}\right)\left(1+\sigma^{-1}\right) \sup _{t \in \mathbb{R}} \int_{t}^{t+1}\|Q(s)\|_{2}^{2} d s .
$$

That is,

$$
\begin{aligned}
\|v(t)\|_{2}^{2} \leq & e^{\sigma(\tau-t)}\left\|v_{0}\right\|_{2}^{2}+2\left(\operatorname{Re}_{1} h^{2}\left(2 \mathrm{Rt}_{2} h^{2}+\frac{2 h}{\alpha}\right)\right) \\
& \times\left(e^{\sigma(\tau-t)}\left\|T_{0}\right\|_{2}^{2}+\rho_{1}\right) .
\end{aligned}
$$

Let $\tau<t-1$, for any $r \in[\tau, t-1]$; integrating (32) from $r$ to $r+1$ and combining with (23), (30), and (37), we obtain

$$
\begin{aligned}
& \int_{r}^{r+1}\|v(s)\|^{2} d s \\
& \leq\|v(r)\|_{2}^{2}+\operatorname{Re}_{1} h^{2} \int_{r}^{r+1}\|T(s)\|_{2}^{2} d s \\
& \leq\|v(r)\|_{2}^{2}+\operatorname{Re}_{1} h^{2}\left(2 \mathrm{Rt}_{2} h^{2}+\frac{2 h}{\alpha}\right) \\
& \quad \times \int_{r}^{r+1}\|T(s)\|^{2} d s \\
& \leq e^{\sigma(\tau-r)}\left\|v_{0}\right\|_{2}^{2}+4\left(\operatorname{Re}_{1} h^{2}\left(2 \mathrm{Rt}_{2} h^{2}+\frac{2 h}{\alpha}\right)\right) \\
& \quad \times\left(e^{\sigma(\tau-r)}\left\|T_{0}\right\|_{2}^{2}+\rho_{1}\right) .
\end{aligned}
$$


3.2.3. $L^{6}(\Omega)$ Estimates of T. Taking the inner product of (2) with $|T|^{4} T$ in $L^{2}(\Omega)$ and using the boundary conditions (4), we have

$$
\begin{gathered}
\frac{1}{6} \frac{d}{d t}\|T\|_{6}^{6}+\left.\left.\frac{5}{9 \mathrm{Rt}_{1}} \int_{\Omega}|\nabla| T\right|^{3}\right|^{2} d x d y d z \\
+\left.\left.\frac{5}{9 \mathrm{Rt}_{2}} \int_{\Omega}\left|\partial_{z}\right| T\right|^{3}\right|^{2} d x d y d z \\
+\alpha \int_{M}|T(z=0)|^{6} d x d y \\
\leq\left\||T|^{3}\right\|_{10 / 3}^{5 / 3}\|Q\|_{2} \\
\leq C\|Q\|_{2}\left\||T|^{3}\right\|_{2}^{2 / 3}\left\||T|^{3}\right\|_{H^{1}(\Omega)} \\
=C\|Q\|_{2}\|T\|_{6}^{2}\left\||T|^{3}\right\|_{H^{1}(\Omega)} .
\end{gathered}
$$

Using the Young inequality, we obtain

$$
\frac{d}{d t}\left(e^{\sigma t}\|T\|_{6}^{2}\right) \leq \sigma e^{\sigma t}\|T\|_{6}^{2}+C e^{\sigma t}\|Q\|_{2}^{2} .
$$

Integrating (40) from $\tau$ to $t$ and combining with (27) and $\|T\|_{6} \leq C\|T\|$, we get

$$
\begin{aligned}
e^{\sigma t}\|T\|_{6}^{2} \leq & e^{\sigma \tau}\left\|T_{0}\right\|_{6}^{2}+\sigma \int_{\tau}^{t} e^{\sigma s}\|T(s)\|_{6}^{2} d s \\
& +C \int_{\tau}^{t} e^{\sigma s}\|Q(s)\|_{2}^{2} d s \\
\leq & e^{\sigma \tau}\left\|T_{0}\right\|^{2}+C \int_{\tau}^{t} e^{\sigma s}\|T(s)\|^{2} d s \\
& +C \int_{\tau}^{t} e^{\sigma s}\|Q(s)\|_{2}^{2} d s \\
\leq & C e^{\sigma \tau}\left\|T_{0}\right\|_{2}^{2}+C e^{\sigma t}\left(2 \mathrm{Rt}_{2} h^{2}+\frac{2 h}{\alpha}+1\right)\left(1+\sigma^{-1}\right) \\
& \times \sup _{t \in \mathbb{R}} \int_{t}^{t+1}\|Q(s)\|_{2}^{2} d s .
\end{aligned}
$$

Therefore, we have

$$
\begin{aligned}
\|T\|_{6}^{2} \leq & C e^{\sigma(\tau-t)}\left\|T_{0}\right\|_{2}^{2} \\
& +C\left(2 \mathrm{Rt}_{2} h^{2}+\frac{2 h}{\alpha}+1\right)\left(1+\sigma^{-1}\right) \\
& \times \sup _{t \in \mathbb{R}} \int_{t}^{t+1}\|Q(s)\|_{2}^{2} d s .
\end{aligned}
$$

3.2.4. $\left(L^{6}(\Omega)\right)^{2}$ Estimates of $\widetilde{v}$. Multiplying (16) by $|\widetilde{v}|^{4} \widetilde{v}$ and integrating by parts over $\Omega$, we deduce

$$
\begin{aligned}
& \frac{1}{6} \frac{d}{d t}\|\widetilde{v}\|_{6}^{6}+\frac{1}{\operatorname{Re}_{1}} \int_{\Omega}|\nabla \widetilde{v}|^{2}|\widetilde{v}|^{4} d x d y d z \\
& \quad+\frac{1}{\operatorname{Re}_{2}} \int_{\Omega}\left|\partial_{z} \widetilde{v}\right|^{2}|\widetilde{v}|^{4} d x d y d z \\
& \quad+\left.\left.\frac{4}{9 \operatorname{Re}_{1}} \int_{\Omega}|\nabla| \widetilde{v}\right|^{3}\right|^{2} d x d y d z \\
& \quad+\left.\left.\frac{4}{9 \operatorname{Re}_{2}} \int_{\Omega}\left|\partial_{z}\right| \widetilde{v}\right|^{3}\right|^{2} d x d y d z \\
& \leq C \int_{\Omega}|\bar{v}||\nabla \widetilde{v}||\widetilde{v}|^{5} d x d y d z \\
& \quad+C \int_{M}\left(\int_{-h}^{0}|T| d z\right)\left(\int_{-h}^{0}|\nabla \widetilde{v}||\widetilde{v}|^{4} d z\right) d x d y \\
& \quad+C \int_{M}\left(\int_{-h}^{0}|\widetilde{v}|^{2} d z\right)\left(\int_{-h}^{0}|\nabla \widetilde{v}||\widetilde{v}|^{4} d z\right) d x d y .
\end{aligned}
$$

It follows from interpolation inequality and Lemma 2 that

$$
\begin{aligned}
& \int_{\Omega}|\bar{v}||\nabla \widetilde{v}||\widetilde{v}|^{5} d x d y d z \\
& \leq \int_{M}|\bar{v}|\left(\int_{-h}^{0}|\nabla \widetilde{v}|^{2}|\widetilde{v}|^{4} d z\right)^{1 / 2}\left(\int_{-h}^{0}|\widetilde{v}|^{6} d z\right)^{1 / 2} d x d y \\
& \leq\left(\int_{M}|\bar{v}|^{4} d x d y\right)^{1 / 4}\left(\int_{\Omega}|\nabla \widetilde{v}|^{2}|\widetilde{v}|^{4} d x d y d z\right)^{1 / 2} \\
& \quad \times\left(\int_{-h}^{0}\left(\int_{M}|\widetilde{v}|^{12} d x d y\right)^{1 / 2} d z\right)^{1 / 2}, \\
& \int_{M}|\widetilde{v}|^{12} d x d y=\left.\left.\int_{M}|| \widetilde{v}\right|^{3}\right|^{4} d x d y \\
& \leq\left.\left. C \int_{M}|\widetilde{|v|}|^{6} d x d y \int_{M}|\nabla| \widetilde{v}\right|^{3}\right|^{2} d x d y,
\end{aligned}
$$

which implies that

$$
\begin{aligned}
& \left(\int_{-h}^{0}\left(\int_{M}|\widetilde{\mid}|^{12} d x d y\right)^{1 / 2} d z\right)^{1 / 2} \\
& \quad \leq C\left(\int_{\Omega}|\widetilde{v}|^{6} d x d y d z\right)^{1 / 4}\left(\left.\left.\int_{\Omega}|\nabla| \widetilde{v}\right|^{3}\right|^{2} d x d y d z\right)^{1 / 4} .
\end{aligned}
$$

We deduce from (44) and (46) that

$$
\begin{aligned}
& \int_{\Omega}|\bar{v}||\nabla \widetilde{v}||\widetilde{v}|^{5} d x d y d z \\
& \leq C\|\widetilde{v}\|_{6}^{3 / 2}\|v\|_{2}^{1 / 2}\|\nabla v\|_{2}^{1 / 2}\left(\left.\left.\int_{\Omega}|\nabla| \widetilde{v}\right|^{3}\right|^{2} d x d y d z\right)^{1 / 4} \\
& \quad \times\left(\int_{\Omega}|\nabla \widetilde{v}|^{2}|\widetilde{v}|^{4} d x d y d z\right)^{1 / 2} .
\end{aligned}
$$


Repeating the similar process with the previously mentioned, we have

$$
\begin{gathered}
\int_{M}\left(\int_{-h}^{0}|T| d z\right)\left(\int_{-h}^{0}|\nabla \widetilde{v}||\widetilde{v}|^{4} d z\right) d x d y \\
\leq C\|T\|_{6}\left(\int_{\Omega}|\nabla \widetilde{v}|^{2}|\widetilde{v}|^{4} d x d y d z\right)^{1 / 2}\|\widetilde{v}\|_{6}^{2}, \\
\int_{M}\left(\int_{-h}^{0}|\widetilde{v}|^{2} d z\right)\left(\int_{-h}^{0}|\nabla \widetilde{v}||\widetilde{v}|^{4} d z\right) d x d y \\
\leq C\left(\int_{\Omega}|\nabla \widetilde{v}|^{2}|\widetilde{v}|^{4} d x d y d z\right)^{1 / 2}\|\widetilde{v}\|_{6}^{3}\|\widetilde{v}\|_{H^{1}(\Omega)},
\end{gathered}
$$

where we use the inequality $\|\widetilde{v}\|_{L^{8}(M)}^{8} \leq C\|\widetilde{v}\|_{L^{6}(M)}^{6}\|\widetilde{v}\|_{H^{1}(M)}^{2}$.

We deduce from (43) and (47)-(48) that

$$
\begin{aligned}
& \frac{d}{d t}\|\widetilde{v}\|_{6}^{6}+\frac{2}{\operatorname{Re}_{1}} \int_{\Omega}|\nabla \widetilde{v}|^{2}|\widetilde{v}|^{4} d x d y d z \\
&+\frac{2}{\operatorname{Re}_{2}} \int_{\Omega}\left|\partial_{z} \widetilde{v}\right|^{2}|\widetilde{v}|^{4} d x d y d z \\
&+\left.\left.\frac{2}{\operatorname{Re}_{1}} \int_{\Omega}|\nabla| \widetilde{v}\right|^{3}\right|^{2} d x d y d z \\
&+\left.\left.\frac{2}{\operatorname{Re}_{2}} \int_{\Omega}\left|\partial_{z}\right| \widetilde{v}\right|^{3}\right|^{2} d x d y d z \\
& \leq C\left(\|v\|_{2}^{2}\|\nabla v\|_{2}^{2}+\|\widetilde{v}\|_{H^{1}(\Omega)}^{2}\right)\|\widetilde{v}\|_{6}^{6} \\
&+C\|T\|_{6}^{2}\|\widetilde{v}\|_{6}^{4},
\end{aligned}
$$

which implies that

$$
\begin{aligned}
& \frac{d}{d t}\left(e^{\sigma t}\|\widetilde{v}\|_{6}^{2}\right) \\
& \leq C e^{\sigma t}\left(1+\|v\|_{2}^{2}\|\nabla v\|_{2}^{2}+\|\widetilde{v}\|_{H^{1}(\Omega)}^{2}\right)\|\widetilde{v}\|_{6}^{2} \\
& \quad+C e^{\sigma t}\|T\|_{6}^{2} .
\end{aligned}
$$

Therefore, it follows from (35)-(38), (42), and Lemma 1 that there exists a positive constant $\rho_{2}, \rho_{3}$ independent of $t$ and a constant $\tau_{1}=\tau_{1}(t)$ such that

$$
\begin{gathered}
\|\widetilde{v}\|_{6}^{2} \leq \rho_{2} \\
\int_{t}^{t+1} e^{\sigma s} \int_{\Omega}|\nabla \widetilde{v}|^{2}|\widetilde{v}|^{4} d x d y d z d s \leq \rho_{3}
\end{gathered}
$$

for any $\tau \leq \tau_{1}$. For brevity, we omit writing out explicitly these bounds here, and we also omit writing out other similar bounds in our future discussion for all other uniform a priori estimates.
3.2.5. $\left(H^{1}(M)\right)^{2}$ Estimates of $\bar{v}$. Taking the $L^{2}(\Omega)$ inner product of (14) with $-\Delta \bar{v}$ and combining the boundary conditions (15), we deduce

$$
\begin{aligned}
& \frac{1}{2} \frac{d}{d t}\|\nabla \bar{v}\|_{L^{2}(M)}^{2}+\frac{1}{\operatorname{Re}_{1}} \int_{M}|\Delta \bar{v}|^{2} d x d y \\
& \leq C \int_{M}|\bar{v}||\nabla \bar{v}||\Delta \bar{v}| d x d y \\
& \quad+C \int_{M}\left(\int_{-h}^{0}|\nabla \bar{v}||\widetilde{v}| d z\right)|\Delta \bar{v}| d x d y \\
& \quad+C \int_{M}|\bar{v}||\Delta \bar{v}| d x d y \\
& \quad+C \int_{M}\left(\int_{-h}^{0}|\nabla T| d z\right)|\Delta \bar{v}| d x d y .
\end{aligned}
$$

We give the estimates of each term of the right-hand side of (53) as follows:

$$
\begin{aligned}
& \int_{M}|\bar{v}||\nabla \bar{v}||\Delta \bar{v}| d x d y \\
& \leq C\left(\int_{M}|\bar{v}|^{4} d x d y\right)^{1 / 4}\left(\int_{M}|\nabla \bar{v}|^{4} d x d y\right)^{1 / 4} \\
& \times\left(\int_{M}|\Delta \bar{v}|^{2} d x d y\right)^{1 / 2} \\
& \leq C\left(\int_{M}|\bar{v}|^{2} d x d y\right)^{1 / 4}\left(\int_{M}|\nabla \bar{v}|^{2} d x d y\right)^{1 / 2} \\
& \times\left(\int_{M}|\Delta \bar{v}|^{2} d x d y\right)^{3 / 4} \\
& \int_{M}\left(\int_{-h}^{0}|\widetilde{v}||\nabla \widetilde{v}| d z\right)|\Delta \bar{v}| d x d y \\
& \leq C\left(\int_{\Omega}|\nabla \widetilde{v}|^{2}|\widetilde{v}|^{4} d x d y d z\right)^{1 / 4}\|\nabla \widetilde{v}\|_{2}^{1 / 2} \\
& \times\left(\int_{M}|\Delta \bar{v}|^{2} d x d y\right)^{1 / 2} \\
& \int_{M}|\bar{v}||\Delta \bar{v}| d x d y \\
& \leq C\left(\int_{M}|\bar{v}|^{2} d x d y\right)^{1 / 2}\left(\int_{M}|\Delta \bar{v}|^{2} d x d y\right)^{1 / 2}, \\
& \int_{M}\left(\int_{-h}^{0}|\nabla T| d z\right)|\Delta \bar{v}| d x d y \\
& \leq C\left(\int_{\Omega}|\nabla T|^{2} d x d y d z\right)^{1 / 2}\left(\int_{M}|\Delta \bar{\nu}|^{2} d x d y\right)^{1 / 2} .
\end{aligned}
$$


It follows from (53)-(54) that

$$
\begin{aligned}
& \frac{d}{d t}\left(e^{\sigma t}\|\nabla \bar{v}\|_{L^{2}(M)}^{2}\right)+\frac{1}{\operatorname{Re}_{1}} e^{\sigma t} \int_{M}|\Delta \bar{v}|^{2} d x d y \\
& \leq C\left(1+\|\bar{v}\|_{L^{2}(M)}^{2}\|\nabla \bar{v}\|_{L^{2}(M)}^{2}\right) e^{\sigma t}\|\nabla \bar{v}\|_{L^{2}(M)}^{2} \\
& \quad+C e^{\sigma t}\|\nabla T\|_{2}^{2}+C e^{\sigma t}\|v\|_{2}^{2} \\
& \quad+C e^{\sigma t} \int_{\Omega}|\nabla \widetilde{v}|^{2}|\widetilde{v}|^{4} d x d y d z+C e^{\sigma t}\|\nabla \widetilde{v}\|_{2}^{2} .
\end{aligned}
$$

In view of (27), (35)-(38), (52), and Lemma 1, we obtain

$$
\|\nabla \bar{v}\|_{L^{2}(M)}^{2} \leq \rho_{4},
$$

for any $\tau \leq \tau_{1}-1$.

3.2.6. $\left(L^{2}(\Omega)\right)^{2}$ Estimates of $v_{z}$. Denote $u=v_{z}$. It is clear that $u$ satisfies the following equation obtained by differentiating (1) with respect to $z$ :

$$
\begin{aligned}
\frac{\partial u}{\partial t}+ & L_{1} u+(v \cdot \nabla) u-\left(\int_{-h}^{z} \nabla \cdot v(x, y, \zeta, t) d \zeta\right) \frac{\partial u}{\partial z} \\
& +(u \cdot \nabla) v-(\nabla \cdot v) u+\frac{1}{\mathrm{Ro}} f u^{\perp}-\nabla T=0,
\end{aligned}
$$

with the boundary conditions

$$
\begin{array}{cc}
\left.u\right|_{z=0}=0, & \left.u\right|_{z=-h}=0, \\
\left.u \cdot \vec{n}\right|_{\Gamma_{l}}=0, & \frac{\partial u}{\partial \vec{n}} \times\left.\vec{n}\right|_{\Gamma_{l}}=0 .
\end{array}
$$

Multiplying (57) by $u$, integrating over $\Omega$, and using the boundary conditions (58), we get

$$
\begin{aligned}
& \frac{1}{2} \frac{d}{d t}\|u\|_{2}^{2}+\frac{1}{\operatorname{Re}_{1}} \int_{\Omega}|\nabla u|^{2} d x d y d z \\
& \quad+\frac{1}{\operatorname{Re}_{2}} \int_{\Omega}\left|\partial_{z} u\right|^{2} d x d y d z \\
& =-\int_{\Omega}[(u \cdot \nabla) v-(\nabla \cdot v) u-\nabla T] \cdot u d x d y d z \\
& \leq C \int_{\Omega}|v||u||\nabla u| d x d y d z+\int_{\Omega}|T||\nabla u| d x d y d z
\end{aligned}
$$

We estimate the right-hand side of (59) term by term as follows:

$$
\begin{gathered}
\int_{\Omega}|T||\nabla u| d x d y d z \leq\|T\|_{2}\|\nabla u\|_{2}, \\
\int_{\Omega}|v||u||\nabla u| d x d y d z \leq\|v\|_{6}\|u\|_{3}\|\nabla u\|_{2} \\
\leq C\|v\|_{6}\|u\|_{2}^{1 / 2}\left(\|\nabla u\|_{2}+\left\|\partial_{z} u\right\|_{2}\right)^{3 / 2} .
\end{gathered}
$$

It follows from (59)-(60) that

$$
\begin{aligned}
& \frac{d}{d t}\|u\|_{2}^{2}+\frac{1}{\operatorname{Re}_{1}} \int_{\Omega}|\nabla u|^{2} d x d y d z+\frac{1}{\operatorname{Re}_{2}} \int_{\Omega}\left|\partial_{z} u\right|^{2} d x d y d z \\
& \quad \leq C\|v\|_{6}^{4}\|u\|_{2}^{2}+C\|T\|_{2}^{2} .
\end{aligned}
$$

Therefore, we have

$$
\begin{gathered}
\frac{d}{d t}\left(e^{\sigma t}\|u\|_{2}^{2}\right)+\frac{1}{\operatorname{Re}_{1}} e^{\sigma t} \int_{\Omega}|\nabla u|^{2} d x d y d z \\
+\frac{1}{\operatorname{Re}_{2}} e^{\sigma t} \int_{\Omega}\left|\partial_{z} u\right|^{2} d x d y d z \\
\leq C\left(1+\|v\|_{6}^{4}\right) e^{\sigma t}\|u\|_{2}^{2}+C e^{\sigma t}\|T\|_{2}^{2} .
\end{gathered}
$$

It is shown in [30] that

$$
\|v\|_{6} \leq C h^{-1 / 3}\|v\|_{2}+C h^{1 / 6}\|\nabla \bar{v}\|_{2}+\|\widetilde{v}\|_{6},
$$

which implies that

$$
\|v\|_{6}^{2} \leq \rho_{5}
$$

for any $\tau \leq \tau_{1}-1$.

By virtue of Lemma 1, from (23), (27)-(29), and (35), we deduce

$$
\begin{gathered}
\left\|\partial_{z} v\right\|_{2}^{2} \leq \rho_{6} \\
\frac{1}{\operatorname{Re}_{1}} \int_{t}^{t+1} \int_{\Omega}\left|\nabla v_{z}\right|^{2} d x d y d z d s \\
+\frac{1}{\operatorname{Re}_{2}} \int_{t}^{t+1} \int_{\Omega}\left|\partial_{z} v_{z}\right|^{2} d x d y d z d s \leq \rho_{7}
\end{gathered}
$$

for any $\tau \leq \tau_{1}-2$.

3.2.7. $\left(L^{2}(\Omega)\right)^{2}$ Estimates of $\nabla v$. Taking the $L^{2}(\Omega)$ inner product of (1) with $-\Delta v$ and combining the boundary conditions (3), we deduce

$$
\begin{aligned}
& \frac{1}{2} \frac{d}{d t}\|\nabla v\|_{2}^{2}+\frac{1}{\operatorname{Re}_{1}} \int_{\Omega}|\Delta v|^{2} d x d y d z \\
& \quad+\frac{1}{\operatorname{Re}_{2}} \int_{\Omega}\left|\nabla \partial_{z} v\right|^{2} d x d y d z \\
& \leq C \int_{M}\left(\int_{-h}^{0}|\nabla v| d z\right)\left(\int_{-h}^{0}\left|\partial_{z} v\right||\Delta v| d z\right) d x d y \\
& \quad+C \int_{M}\left(\int_{-h}^{0}|\nabla T| d z\right)\left(\int_{-h}^{0}|\Delta v| d z\right) d x d y \\
& \quad+C \int_{\Omega}|v||\nabla v||\Delta v| d x d y d z \\
& \quad+C \int_{\Omega}|v||\Delta v| d x d y d z .
\end{aligned}
$$


We estimate each term in the right-hand side of (67) as follows

$$
\begin{gathered}
\int_{\Omega}|v||\nabla v||\Delta v| d x d y d z \\
\leq C\|v\|_{6}\|\nabla v\|_{3}\|\Delta v\|_{2} \\
\leq C\|v\|_{6}\|\nabla v\|_{2}^{1 / 2}\left(\left\|\nabla \partial_{z} v\right\|_{2}+\|\Delta v\|_{2}\right)^{3 / 2}, \\
\int_{M}\left(\int_{-h}^{0}|\nabla v| d z\right)\left(\int_{-h}^{0}\left|\partial_{z} v\right||\Delta v| d z\right) d x d y \\
\leq C\left\|v_{z}\right\|_{2}^{1 / 2}\left\|\nabla v_{z}\right\|_{2}^{1 / 2}\|\nabla v\|_{2}^{1 / 2}\|\Delta v\|_{2}^{3 / 2}, \\
\int_{M}\left(\int_{-h}^{0}|\nabla T| d z\right)\left(\int_{-h}^{0}|\Delta v| d z\right) d x d y \leq C\|\nabla T\|_{2}\|\Delta v\|_{2}, \\
\int_{\Omega}|v||\Delta v| d x d y d z \leq C\|v\|_{2}\|\Delta v\|_{2} .
\end{gathered}
$$

We derive from (67)-(68) that

$$
\begin{gathered}
\frac{d}{d t}\|\nabla v\|_{2}^{2}+\frac{1}{\operatorname{Re}_{1}} \int_{\Omega}|\Delta v|^{2} d x d y d z \\
+\frac{1}{\operatorname{Re}_{2}} \int_{\Omega}\left|\nabla \partial_{z} v\right|^{2} d x d y d z \\
\leq C\left(\|v\|_{6}^{4}+\left\|\partial_{z} v\right\|_{2}^{2}\left\|\nabla \partial_{z} v\right\|_{2}^{2}\right)\|\nabla v\|_{2}^{2} \\
+C\|\nabla T\|_{2}^{2}+C\|v\|_{2}^{2} .
\end{gathered}
$$

Therefore, we have

$$
\begin{aligned}
& \frac{d}{d t}\left(e^{\sigma t}\|\nabla v\|_{2}^{2}\right)+\frac{1}{\operatorname{Re}_{1}} e^{\sigma t} \int_{\Omega}|\Delta v|^{2} d x d y d z \\
& +\frac{1}{\operatorname{Re}_{2}} e^{\sigma t} \int_{\Omega}\left|\nabla \partial_{z} v\right|^{2} d x d y d z \\
& \leq C\left(1+\|v\|_{6}^{4}+\left\|\partial_{z} v\right\|_{2}^{2}\left\|\nabla \partial_{z} v\right\|_{2}^{2}\right) e^{\sigma t}\|\nabla v\|_{2}^{2} \\
& +C e^{\sigma t}\|\nabla T\|_{2}^{2}+C e^{\sigma t}\|v\|_{2}^{2} .
\end{aligned}
$$

By virtue of (27), (35), (64)-(66), and Lemma 1, we get

$$
\begin{gathered}
\|\nabla v\|_{2}^{2} \leq \rho_{8} \\
\frac{1}{\operatorname{Re}_{1}} \int_{t}^{t+1} \int_{\Omega}|\Delta v|^{2} d x d y d z d \tau \\
+\frac{1}{\operatorname{Re}_{2}} \int_{t}^{t+1} \int_{\Omega}\left|\nabla \partial_{z} v\right|^{2} d x d y d z d \tau \leq \rho_{9},
\end{gathered}
$$

for any $\tau \leq \tau_{1}-3$.
3.2.8. $H^{1}(\Omega)$ Estimates of $T$. Multiplying (2) by $L_{2} T$, integrating over $\Omega$, and combining the boundary conditions (4), we deduce

$$
\begin{aligned}
& \frac{1}{2} \frac{d}{d t}\|T\|^{2}+\left\|L_{2} T\right\|_{2}^{2} \\
& \leq C \int_{\Omega}|v||\nabla T|\left|L_{2} T\right| \\
& \quad+C \int_{\Omega}\left(\int_{-h}^{z} \nabla \cdot v(x, y, \zeta, t) d \zeta\right)\left|\frac{\partial T}{\partial z}\right|\left|L_{2} T\right| \\
& \quad+\int_{\Omega}|Q|\left|L_{2} T\right| .
\end{aligned}
$$

We give the estimates of each term in the right-hand side of (73) as follows

$$
\begin{gathered}
\int_{\Omega}|v||\nabla T|\left|L_{2} T\right| d x d y d z \leq\|v\|_{6}\|\nabla T\|_{3}\left\|L_{2} T\right\|_{2} \\
\leq C\|v\|_{6}\|\nabla T\|_{2}^{1 / 2}\left\|L_{2} T\right\|_{2}^{3 / 2} \\
\int_{M}\left(\int_{-h}^{0}|\nabla v| d z\right)\left(\int_{-h}^{0}\left|\partial_{z} T\right|\left|L_{2} T\right| d z\right) d x d y \\
\leq C\|\nabla v\|_{2}^{1 / 2}\|\Delta v\|_{2}^{1 / 2}\left\|\partial_{z} T\right\|_{2}^{1 / 2}\left\|L_{2} T\right\|_{2}^{3 / 2} \\
\int_{\Omega}|Q|\left|L_{2} T\right| d x d y d z \leq C\|Q\|_{2}\left\|L_{2} T\right\|_{2} .
\end{gathered}
$$

We derive from (73)-(74) that

$$
\begin{aligned}
& \frac{d}{d t}\left(e^{\sigma t}\|T\|^{2}\right) \\
& \leq C\left(1+\|v\|_{6}^{4}+\|\nabla v\|_{2}^{2}\|\Delta v\|_{2}^{2}\right) e^{\sigma t}\|T\|^{2} \\
& \quad+C e^{\sigma t}\|Q\|_{2}^{2} .
\end{aligned}
$$

Using (27), (64), (71), (72), and Lemma 1, we get

$$
\begin{gathered}
\|T\|^{2} \leq \rho_{10}, \\
\int_{t}^{t+1}\left\|L_{2} T(s)\right\|_{2}^{2} d s \leq \rho_{11},
\end{gathered}
$$

for any $\tau \leq \tau_{1}-4$.

\section{The Existence of the Pullback Attractor}

In this section, we recall some definitions and lemmas about pullback attractor and prove the existence of $(V, V)$-pullback attractor.

Definition 4 (see $[13,32])$. Let $X$ be a complete metric space. A two-parameter family of mappings $\{U(t, \tau)\}_{\tau \leq t}$ is said to be a continuous process in $X$ if

(i) $U(\tau, \tau)=$ Id for any $\tau \in \mathbb{R}$,

(ii) $U(t, r) U(r, \tau)=U(t, \tau), t \geq r \geq \tau$,

(iii) $U(t, \tau) x_{n} \rightarrow U(t, \tau) x$, if $x_{n} \rightarrow x$ in $X$. 
Definition 5 (see $[11,13,33])$. Let $X, Y$ be two Banach spaces. Then, the family $\widehat{\mathscr{A}}=\{A(t): t \in \mathbb{R}\}$ is said to be a $(X, Y)$-pullback attractor for $\{U(t, \tau)\}_{t \geq \tau}$, if

(i) $A(t)$ is closed in $X$ and compact in $Y$ for any $t \in \mathbb{R}$,

(ii) $U(t, \tau) A(\tau)=A(t)$ for any $\tau \leq t$ (invariance property),

(iii) it pullback attracts every bounded subsets of $X$ in the topology of $Y((X, Y)$-pullback attracting), that is, for any nonempty class of bounded subsets $\{D(t): t \in$ $\mathbb{R}\} \subset X$,

$$
\lim _{\tau \rightarrow-\infty} \operatorname{dist}_{Y}(U(t, \tau) D(\tau), A(t))=0,
$$

for any $t \in \mathbb{R}$.

Definition 6 (see $[31,33]$ ). The process $\{U(t, \tau)\}_{t \geq \tau}$ is said to be pullback asymptotically compact if for any $t \in \mathbb{R}$ and any nonempty class of bounded subsets $\{D(t): t \in \mathbb{R}\} \subset X$, any sequence $\tau_{n} \rightarrow-\infty$, and any sequence $x_{n} \in D\left(\tau_{n}\right)$, the sequence $\left\{U\left(t, \tau_{n}\right) x_{n}\right\}$ is relatively compact in $X$.

Lemma 7 (see [33]). Let $\{U(t, \tau)\}_{t \geq \tau}$ be a continuous process such that $\{U(t, \tau)\}$ is pullback asymptotically compact. If there exists a family of pullback absorbing sets $\widehat{B}=\{B(t): t \in \mathbb{R}\}$, then $\{U(t, \tau)\}_{t \geq \tau}$ has a unique pullback attractor $\widehat{\mathscr{A}}=\{\mathscr{A}(t)$ : $t \in \mathbb{R}\}$ and

$$
\mathscr{A}(t)=\bigcap_{s \leq t} \overline{\bigcup_{\tau \leq s}} U(t, \tau) B(\tau) .
$$

Definition 8 (see [33]). A process $\{U(t, \tau)\}_{t \geq \tau}$ is said to satisfy pullback $\mathscr{D}$ condition (PDC), if for any fixed $t \in \mathbb{R}$, any bounded subset $D$ of a Banach space $X$ and any $\eta>0$, there exist a time $\tau_{0}=\tau_{0}(D, \eta, t) \leq t$ and a finite-dimensional subspace $X_{1}$ of $X$ such that

(i) $P\left(\bigcup_{\tau \leq \tau_{0}} U(t, \tau) D\right)$ is bounded,

(ii) (Id $-P) y \leq \eta$ for any $y \in \bigcup_{\tau \leq \tau_{0}} U(t, \tau) x$ and any $x \in D$,

where $P: X \rightarrow X_{1}$ is a bounded projection and Id is the identity.

Lemma 9 (see [33]). A process $\{U(t, \tau)\}_{t \geq \tau}$ satisfying pullback D condition (PDC) is pullback asymptotically compact.

From (29), (37), (65), (71), and (76), we get the following theorem.

Theorem 10. Assume that $Q \in L_{\text {loc }}^{2}\left(\mathbb{R} ; L^{2}(\Omega)\right)$ satisfies

$$
\sup _{t \in \mathbb{R}} \int_{t}^{t+1}\|Q(s)\|_{2}^{2} d s<\infty .
$$

Then, for any $0<\sigma \leq \min \left\{1 /\left(4 R t_{2} h^{2}+(4 h / \alpha)\right), 1 / 2 C_{M} R e_{1}\right\}$, there exists a $(V, V)$-pullback absorbing set for the processes $\{U(t, \tau)\}_{t \geq \tau}$ associated with (1)-(5).
Thanks to the knowledge of functional analysis, we know that the operator $A=\left(L_{1}, L_{2}\right): V \rightarrow V^{\prime}$ with domain

$$
\mathscr{D}(A)=V \cap\left(H^{2}(\Omega)\right)^{3}
$$

is a positive self-adjoint operator with compact inverse. Therefore, the space $H$ possesses an orthonormal basis $\left\{\left(\omega_{k}, \eta_{k}\right)\right\}_{k=1}^{\infty}$ of eigenfunctions of the operator $A$ such that

$$
\left(L_{1} \omega_{k}, L_{2} \eta_{k}\right)=\left(\lambda_{k} \omega_{k}, \mu_{k} \eta_{k}\right) \text {, }
$$

where

$$
\begin{gathered}
0<\lambda_{1} \leq \lambda_{2} \leq \cdots, \\
\lim _{k \rightarrow \infty} \lambda_{k}=\infty, \\
0<\mu_{1} \leq \mu_{2} \leq \cdots, \\
\lim _{k \rightarrow \infty} \mu_{k}=\infty .
\end{gathered}
$$

Let $H_{m}=\operatorname{span}\left\{\left(\omega_{1}, \eta_{1}\right),\left(\omega_{2}, \eta_{2}\right), \ldots,\left(\omega_{m}, \eta_{m}\right)\right\}$, and let $P_{m}$ : $H \rightarrow H_{m}$ be the $L^{2}(\Omega)$ orthogonal projection onto $H_{m}$.

In the following, we prove that the process $\{U(t, \tau)\}_{t \geq \tau}$ associated with (1)-(5) is pullback asymptotically compact in $V$ by verifying the pullback $\mathscr{D}$ condition.

Theorem 11. Assume that $Q \in L_{\text {loc }}^{2}\left(\mathbb{R} ; L^{2}(\Omega)\right)$ satisfies

$$
\sup _{t \in \mathbb{R}} \int_{t}^{t+1}\|Q(s)\|_{2}^{2} d s<\infty .
$$

Then, for any $0<\sigma \leq \min \left\{1 /\left(4 R t_{2} h^{2}+(4 h / \alpha)\right), 1 / 2 C_{M} R e_{1}\right\}$, the process $\{U(t, \tau)\}_{t \geq \tau}$ associated with (1)-(5) is pullback asymptotically compact in $V$.

Proof. Let $B$ be a bounded subset of $H(\Omega),(v(t), T(t))=$ $U(t, \tau)\left(v_{\tau}, T_{\tau}\right)=\left(v_{1}+v_{2}, T_{1}+T_{2}\right)$, where $\left(v_{\tau}, T_{\tau}\right) \in$ $B,\left(v_{1}, T_{1}\right)=P_{m}(v, T)$, and $\left(v_{2}, T_{2}\right)=\left(\operatorname{Id}-P_{m}\right)(v, T)=$ $Q_{m}(v, T)$.

Taking the inner product of (1) with $L_{1} v_{2}$, we have

$$
\begin{aligned}
& \frac{1}{2} \frac{d}{d t}\left\|v_{2}\right\|^{2}+\left\|L_{1} v_{2}\right\|_{2}^{2} \\
& \leq\|v\|_{6}\|\nabla v\|_{3}\left\|L_{1} v_{2}\right\|_{2} \\
& \quad+C\|\nabla v\|_{2}^{1 / 2}\|\Delta v\|_{2}^{1 / 2}\left\|\frac{\partial v}{\partial z}\right\|_{2}^{1 / 2}\left\|\nabla v_{z}\right\|_{2}^{1 / 2}\left\|L_{1} v_{2}\right\|_{2} \\
& \quad+C\|\nabla T\|_{2}\left\|L_{1} v_{2}\right\|_{2}+C\|v\|_{2}\left\|L_{1} v_{2}\right\|_{2} .
\end{aligned}
$$

From the Young inequality, we obtain

$$
\begin{aligned}
\frac{d}{d t}\left\|v_{2}\right\|^{2}+\lambda_{m}\left\|v_{2}\right\|^{2} \leq & C\|v\|_{6}^{2}\|\nabla v\|_{3}^{2}+C\|\nabla v\|_{2}^{2}\|\Delta v\|_{2}^{2} \\
& +C\left\|v_{z}\right\|_{2}^{2}\left\|\nabla v_{z}\right\|_{2}^{2} \\
& +C\|\nabla T\|_{2}^{2}+C\|v\|_{2}^{2} .
\end{aligned}
$$


By virtue of Lemma 1, we get

$$
\left\|v_{2}(t+r)\right\|^{2} \leq e^{-\lambda_{m}}\left(\int_{t}^{t+1}\left\|v_{2}(s)\right\|^{2} d s+\int_{t}^{t+1} b(s) d s\right),
$$

where

$$
\begin{aligned}
b(s)= & C\|v\|_{6}^{2}\|\nabla v\|_{3}^{2}+C\|\nabla v\|_{2}^{2}\|\Delta v\|_{2}^{2} \\
& +C\left\|v_{z}\right\|_{2}^{2}\left\|\nabla v_{z}\right\|_{2}^{2} \\
& +C\|\nabla T\|_{2}^{2}+C\|v\|_{2}^{2} .
\end{aligned}
$$

Therefore, we obtain

$$
\left\|v_{2}(t)\right\|^{2} \leq \frac{\epsilon}{2}
$$

for any $\epsilon>0, t \in \mathbb{R}$, and any $\tau \leq \tau_{2}-1$, if $m_{1}$ is sufficiently large.

Similarly, we get

$$
\left\|T_{2}(t)\right\|^{2} \leq \frac{\epsilon}{2}
$$

for any $\epsilon>0, t \in \mathbb{R}$, and any $\tau \leq \tau_{2}-2$, if $m_{2}$ is sufficiently large. Hence,

$$
\left\|v_{2}\right\|^{2}+\left\|T_{2}(t)\right\|^{2} \leq \epsilon,
$$

for any $\epsilon>0, t \in \mathbb{R}$, and any $\tau \leq \tau_{2}-2$, if $m \geq \max \left\{m_{1}, m_{2}\right\}$.

From Theorems 10 and 11 and Lemma 7, we obtain the following main Theorem.

Theorem 12. Assume that $Q \in L_{\text {loc }}^{2}\left(\mathbb{R} ; L^{2}(\Omega)\right)$ satisfies

$$
\sup _{t \in \mathbb{R}} \int_{t}^{t+1}\|Q(s)\|_{2}^{2} d s<\infty
$$

Then, for any $0<\sigma \leq \min \left\{1 /\left(4 R t_{2} h^{2}+(4 h / \alpha)\right), 1 / 2 C_{M} R e_{1}\right\}$, the process $\{U(t, \tau)\}_{t \geq \tau}$ associated with the solutions of (1)-(5) possesses a $(V, V)$-pullback attractor $\widehat{\mathscr{A}}=\{\mathscr{A}(t): t \in \mathbb{R}\}$.

\section{Acknowledgment}

The authors of this paper would like to express their sincere thanks to the reviewer for valuable comments and suggestions.

\section{References}

[1] C. S. Cao and E. S. Titi, "Global well-posedness and finitedimensional global attractor for a 3-D planetary geostrophic viscous model," Communications on Pure and Applied Mathematics, vol. 56, no. 2, pp. 198-233, 2003.

[2] C. S. Cao, E. S. Titi, and M. Ziane, "A 'horizontal' hyperdiffusion three-dimensional thermocline planetary geostrophic model: well-posedness and long-time behaviour," Nonlinearity, vol. 17, no. 5, pp. 1749-1776, 2004.
[3] D. N. Cheban, P. E. Kloeden, and B. Schmalfuß, "The relationship between pullback, forwards and global attractors of nonautonomous dynamical systems," Nonlinear Dynamics and Systems Theory, vol. 2, pp. 9-28, 2002.

[4] H. Crauel and F. Flandoli, "Attractors for random dynamical systems," Probability Theory and Related Fields, vol. 100, no. 3, pp. 365-393, 1994.

[5] H. Crauel, A. Debussche, and F. Flandoli, "Random attractors," Journal of Dynamics and Differential Equations, vol. 9, no. 2, pp. 307-341, 1997.

[6] T. Caraballo, G. Łukaszewicz, and J. Real, "Pullback attractors for asymptotically compact non-autonomous dynamical systems," Nonlinear Analysis: Theory, Methods \& Applications, vol. 64, no. 3, pp. 484-498, 2006.

[7] P. E. Kloeden and B. Schmalfuß, "Nonautonomous systems, cocycle attractors and variable time-step discretization," Numerical Algorithms, vol. 14, no. 1-3, pp. 141-152, 1997.

[8] P. E. Kloeden and D. J. Stonier, "Cocycle attractors in nonautonomously perturbed differential equations," Dynamics of Continuous, Discrete and Impulsive Systems, vol. 4, no. 2, pp. 211226, 1998.

[9] G. Łukaszewicz, "On pullback attractors in $H_{0}^{1}$ for nonautonomous reaction-diffusion equations," International Journal of Bifurcation and Chaos, vol. 20, no. 9, pp. 2637-2644, 2010.

[10] S. S. Lu, H. Q. Wu, and C. K. Zhong, "Attractors for nonautonomous 2D Navier-Stokes equations with normal external forces," Discrete and Continuous Dynamical Systems A, vol. 13, no. 3, pp. 701-719, 2005.

[11] B. Schmalfuß, "Attractors for non-autonomous dynamical systems," in Proceedings of the International Conference on Differential Equations (Equadiff'99), B. Fiedler, K. Gröer, and J. Sprekels, Eds., pp. 684-689, World Scientific, Berlin, Germany, 2000.

[12] L. Yang, M. H. Yang, and P. E. Kloeden, "Pullback attractors for non-autonomous quasi-linear parabolic equations with dynamical boundary conditions," Discrete and Continuous Dynamical Systems B, vol. 17, no. 7, pp. 2635-2651, 2012.

[13] V. V. Chepyzhov and M. I. Vishik, Attractors for Equations of Mathematical Physics, vol. 49 of American Mathematical Society Colloquium Publications, American Mathematical Society, Providence, RI, USA, 2002.

[14] C. S. Cao and E. S. Titi, "Global well-posedness of the threedimensional viscous primitive equations of large scale ocean and atmosphere dynamics," Annals of Mathematics, vol. 166, no. 1, pp. 245-267, 2007.

[15] J. L. Lions, R. Temam, and S. Wang, "New formulations of the primitive equations of atmosphere and applications," Nonlinearity, vol. 5, no. 2, pp. 237-288, 1992.

[16] J. L. Lions, R. Temam, and S. Wang, "On the equations of the large-scale ocean," Nonlinearity, vol. 5, no. 5, pp. 1007-1053, 1992.

[17] J. Pedlosky, Geophysical Fluid Dynamics, Springer, New York, NY, USA, 1987.

[18] G. K. Vallis, Atmospheric and Oceanic Fluid Dynamics: Fundamentals and Large-Scale Circulation, Cambridge University Press, Cambridge, UK, 2006.

[19] B. D. Ewald and R. Témam, "Maximum principles for the primitive equations of the atmosphere," Discrete and Continuous Dynamical Systems A, vol. 7, no. 2, pp. 343-362, 2001.

[20] B. L. Guo and D. W. Huang, "On the existence of atmospheric attractors," Science in China D, vol. 51, no. 3, pp. 469-480, 2008. 
[21] B. L. Guo and D. W. Huang, "On the 3D viscous primitive equations of the large-scale atmosphere," Acta Mathematica Scientia B, vol. 29, no. 4, pp. 846-866, 2009.

[22] B. L. Guo and D. W. Huang, "Existence of weak solutions and trajectory attractors for the moist atmospheric equations in geophysics," Journal of Mathematical Physics, vol. 47, no. 8, Article ID 083508, 23 pages, 2006.

[23] C. Hu, R. Temam, and M. Ziane, "The primitive equations on the large scale ocean under the small depth hypothesis," Discrete and Continuous Dynamical Systems A, vol. 9, no. 1, pp. 97-131, 2003.

[24] C. Hu, "Asymptotic analysis of the primitive equations under the small depth assumption," Nonlinear Analysis: Theory, Methods \& Applications, vol. 61, no. 3, pp. 425-460, 2005.

[25] M. Petcu, R. Temam, and D. Wirosoetisno, "Existence and regularity results for the primitive equations in two space dimensions," Communications on Pure and Applied Analysis, vol. 3, no. 1, pp. 115-131, 2004.

[26] T. Tachim Medjo, "On the uniqueness of $z$-weak solutions of the three-dimensional primitive equations of the ocean," Nonlinear Analysis: Real World Applications, vol. 11, no. 3, pp. 1413-1421, 2010.

[27] F. Guillén-González, N. Masmoudi, and M. A. RodríguezBellido, "Anisotropic estimates and strong solutions of the primitive equations," Differential and Integral Equations, vol. 14, no. 11, pp. 1381-1408, 2001.

[28] R. Temam and M. Ziane, "Some mathematical problems in geophysical fluid dynamics," in Handbook of Mathematical Fluid Dynamics, vol. 3, pp. 535-658, 2005.

[29] B. You and S. Ma, "Global attractors for three dimensional viscous primitive equations of large-scale atmosphere in logpressure coordinate," Abstract and Applied Analysis, vol. 2013, Article ID 758730, 16 pages, 2013.

[30] N. Ju, "The global attractor for the solutions to the 3D viscous primitive equations," Discrete and Continuous Dynamical Systems A, vol. 17, no. 1, pp. 159-179, 2007.

[31] R. Temam, Infinite-Dimensional Dynamical Systems in Mechanics and Physics, Springer, New York, NY, USA, 1997.

[32] Y. Li and C. K. Zhong, "Pullback attractors for the normto-weak continuous process and application to the nonautonomous reaction-diffusion equations," Applied Mathematics and Computation, vol. 190, no. 2, pp. 1020-1029, 2007.

[33] Y. Wang and C. Zhong, "Pullback D-attractors for nonautonomous Sine-Gordon equations," Nonlinear Analysis: Theory, Methods \& Applications, vol. 67, no. 7, pp. 2137-2148, 2007. 


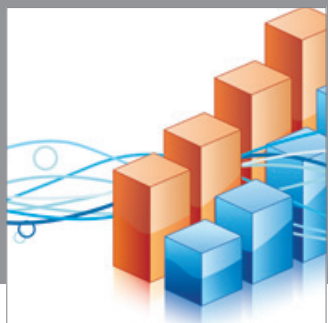

Advances in

Operations Research

mansans

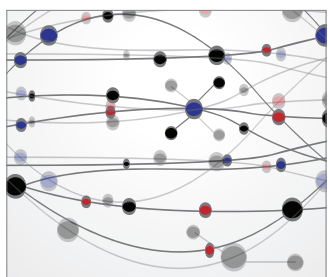

The Scientific World Journal
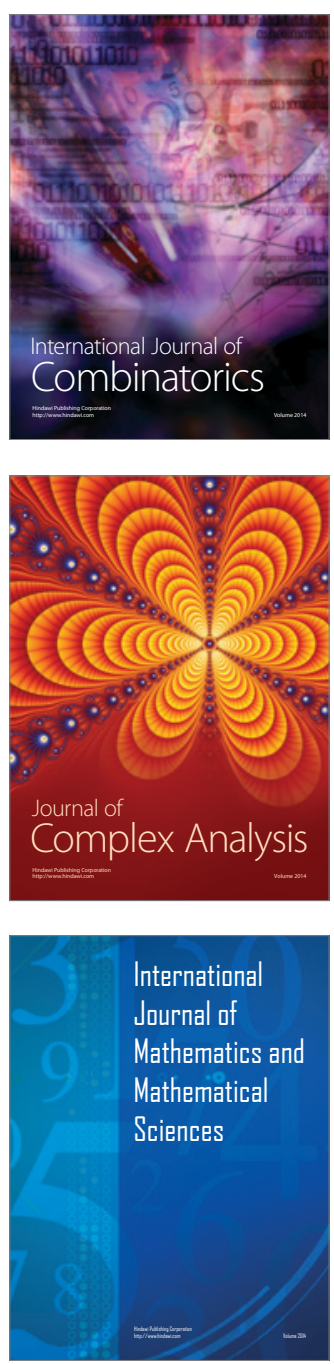
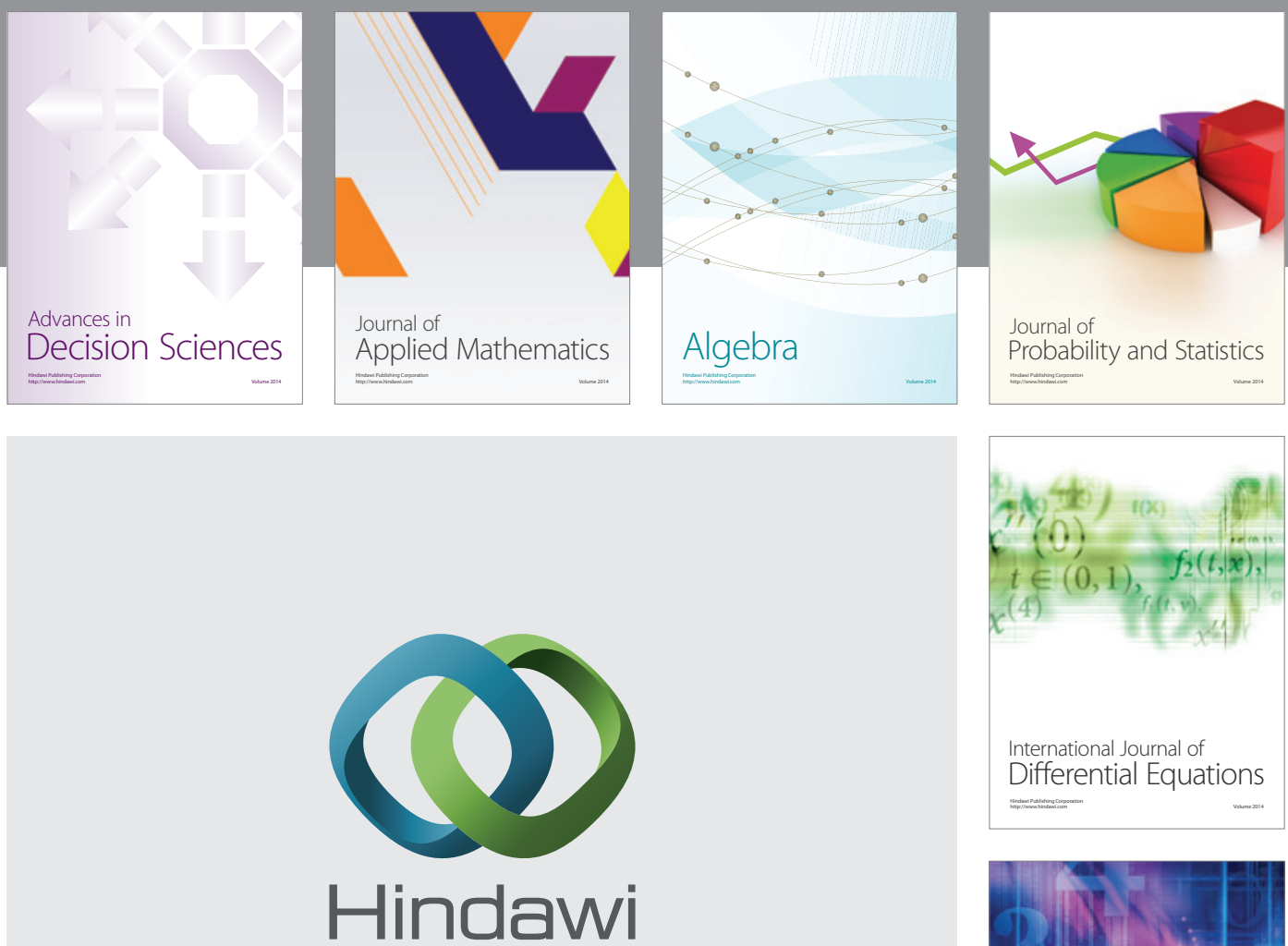

Submit your manuscripts at http://www.hindawi.com
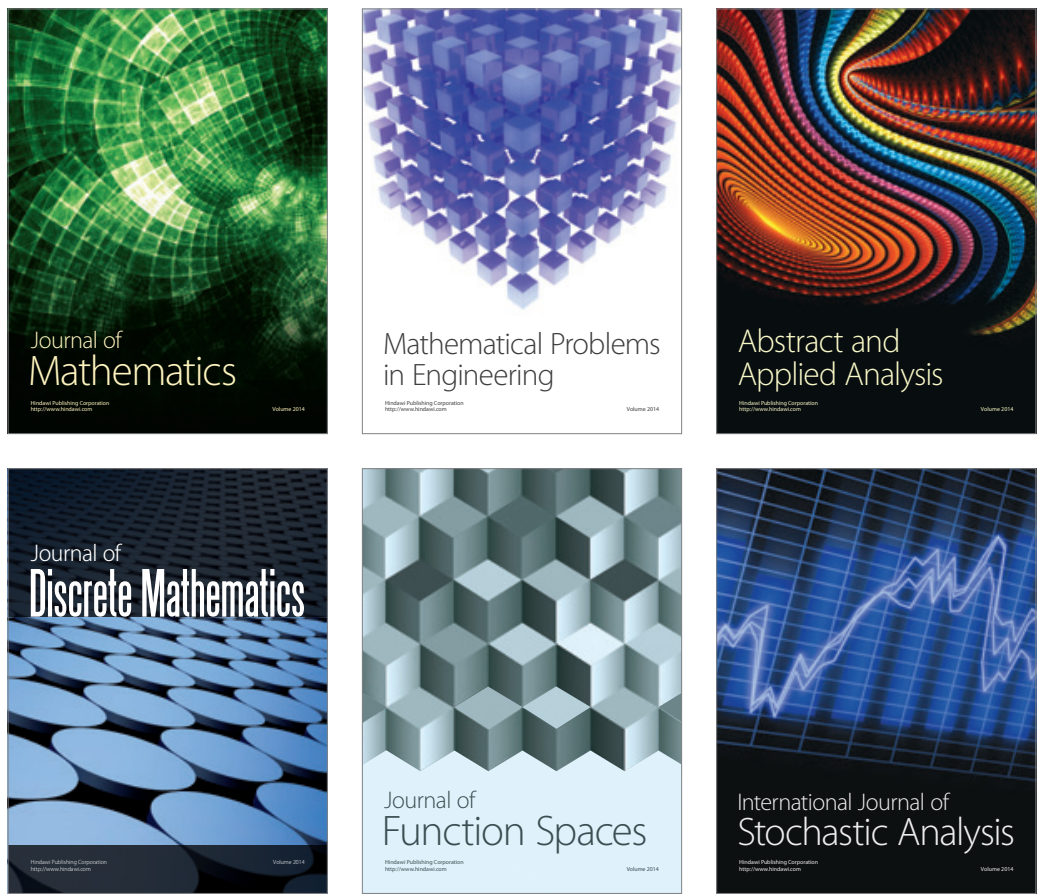

Journal of

Function Spaces

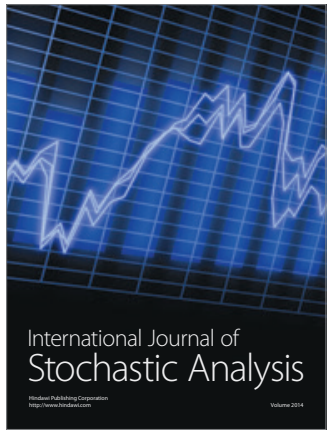

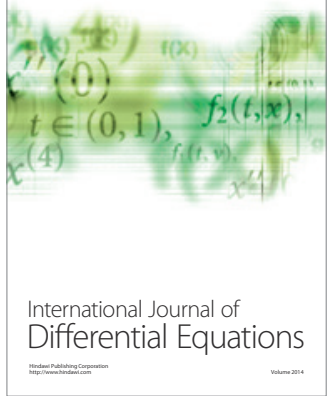
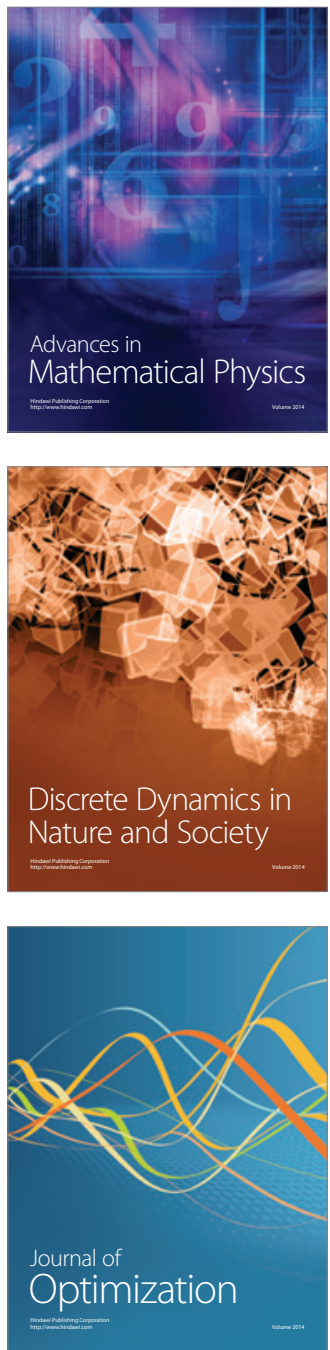